\title{
BMJ Open Protocol for the Sri Lankan Cerebral Palsy Register pilot study
}

Thilini Madushika Heiyanthuduwage (D) , ${ }^{1}$ Samanmali P Sumanasena, ${ }^{1}$ Gopi Kitnasamy, ${ }^{2}$ Hayley Smithers Sheedy, ${ }^{3}$ Gulam Khandaker, ${ }^{4,5,6,7}$ Romaniya Fernando, ${ }^{1}$ Saraji Wijesekara, ${ }^{8}$ Jayatri Jagoda, ${ }^{9}$ Pyara Ratnayake, ${ }^{9}$ Jithangi Wanigasinghe, ${ }^{10}$ Sarah Mclntyre, ${ }^{3}$ Shona Goldsmith, ${ }^{3}$ Emma Waight, ${ }^{3}$ Nadia Badawi, ${ }^{3,11}$ Mohammad Muhit, ${ }^{4,5}$ Nimisha Muttiah ${ }^{12}$

To cite: Heiyanthuduwage TM, Sumanasena SP, Kitnasamy G, et al. Protocol for the Sri Lankan Cerebral Palsy Register pilot study. BMJ Open 2020;10:e039353. doi:10.1136/ bmjopen-2020-039353

- Prepublication history and additional material for this paper are available online. To view these files, please visit the journal online (http://dx.doi. org/10.1136/bmjopen-2020039353).

Received 14 April 2020 Revised 01 July 2020 Accepted 07 July 2020

\section{Check for updates}

(C) Author(s) (or their employer(s)) 2020. Re-use permitted under CC BY-NC. No commercial re-use. See rights and permissions. Published by BMJ.

For numbered affiliations see end of article.

Correspondence to Thilini Madushika

Heiyanthuduwage;

htmadushika@gmail.com

\section{ABSTRACT}

Introduction Cerebral palsy (CP) describes a heterogeneous group of motor disorders resulting from disturbance in the developing brain. CP occurs in approximately 2.1 per 1000 live births in high-income countries, but in low- and middle-income countries (LMICs) the prevalence and severity of CP may be greater and aetiological risk factors different. In Sri Lanka, a LMIC, there have been no epidemiological studies of $\mathrm{CP}$ to date. Systematically collected data are required to identify opportunities for primary and secondary prevention, to plan and establish services to support children and adults with $\mathrm{CP}$ and their families and to act as a sampling frame for new research. Here we describe a pilot study protocol for a CP register in Sri Lanka.

Methods and analysis The aim of this study is to establish a CP register in Sri Lanka. We will use different surveillance methodologies in two provinces of Sri Lanka: hospital and community surveillance in the Western Province and community surveillance in the Eastern Province. A common record form will collect demographic, clinical and service data for children with $\mathrm{CP}<18$ years living in these two provinces. Data will be transferred to a secure online data repository and used to describe the epidemiology of $\mathrm{CP}$ in these regions. We will describe the strengths and challenges of the surveillance mechanisms and estimate the resources required for ongoing hospital and community based surveillance in the Western and Eastern provinces and to include additional provinces across the country.

Ethics and dissemination This study has ethical clearance from The University of Kelaniya, National Health Research Council, the Institutional Ethics Review Committee of the Lady Ridgeway Hospital, Colombo South Teaching Hospital and the Director of the North Colombo Teaching Hospital. Results from this research will be disseminated through local and international conferences and through publications in peer-reviewed journals.

\section{INTRODUCTION}

Cerebral palsy (CP) describes a group of motor disorders which are lifelong and for which there is no cure. ${ }^{1}$ The disorders of movement and posture that are included within this umbrella term of CP are caused by injury, insult or maldevelopment of the

\section{Strengths and limitations of this study}

- This is a protocol for a pilot study to establish a Sri Lankan Cerebral Palsy Register and to describe the epidemiology, demographics and access to rehabilitation services of children with cerebral palsy (CP) in Sri Lanka.

- In this pilot study, data will only be collected from two of the nine provinces. This will limit our ability to extrapolate our findings for all children with CP in Sri Lanka, however this preliminary phase is an important first step towards expanding this work into a national CP surveillance programme.

- Data will be collected via two mechanisms, community and hospital based surveillance, increasing the likelihood of complete or near complete ascertainment. Piloting both methods will also allow us to describe the strengths and challenges of these two different surveillance mechanisms in the Sri Lankan context.

- Initial findings from this pilot study will inform future service planning and support for children with CP and their families in Sri Lanka.

developing brain either in the prenatal/perinatal period or in the post neonatal period (28 days to 2 years of age). ${ }^{12}$ In addition to the motor disorders of $\mathrm{CP}$, associated comorbidities are common including disturbances of sensation and cognition and also the presence of epilepsy. ${ }^{3}$ The rate of CP internationally is estimated to be 2.1 per 1000 live births. ${ }^{4}$ However this estimate is drawn largely from data from high-income countries. The rate of CP in low- and middle-income countries (LMICs) has been estimated to be higher. ${ }^{56}$ Findings from the Bangladesh Cerebral Palsy Register suggest that the prevalence of CP in rural Bangladesh is 3.4 per 1000 children (95\% CI 3.2 to 3.7$){ }^{5}$ In this study proportionally more children had severe functional gross motor limitations $(\approx 50 \%$ of children who required a wheelchair for mobility) 
compared with children with CP in high-income settings such as Australia $(\approx 25 \%){ }^{7}$

Sri Lanka is a LMIC which has free health and education services. The majority of infants are born in hospitals and Sri Lanka has low rates of infant mortality compared with other countries in the region. ${ }^{8}$ However, the epidemiology of CP in Sri Lanka is unknown. In the absence of systematically collected data on the prevalence, aetiology, risk factors, clinical features (eg, CP motor type, motor function, associated impairments) of children with CP, it is challenging to develop appropriate support services for this most vulnerable population. In a LMIC setting like Sri Lanka where services for people with disabilities are limited this is particularly important. CP registers are useful for informing planning and can also provide a sampling frame for future research. Here we describe the protocol for the pilot Sri Lankan CP Register.

\section{Aims and objectives}

Our overarching aim is to develop a platform for a national CP register in Sri Lanka to facilitate research on the prevalence, aetiology, risk factors, motor type and severity and associated impairments of children with CP in Sri Lanka. Importantly register data will be used to understand the clinical profile of CP in Sri Lanka to inform rehabilitation service planning. This is a collaborative project between the University of Kelaniya (UKel) and the Cerebral Palsy Lanka Foundation (CPLF) from Sri Lanka and the Cerebral Palsy Alliance in Australia.

Our specific objectives for this pilot study are to:

1. Develop and establish the Sri Lankan CP Register.

a. Develop a core data set of items that will be collected in the Western and Eastern provinces (figure 1) of Sri Lanka.

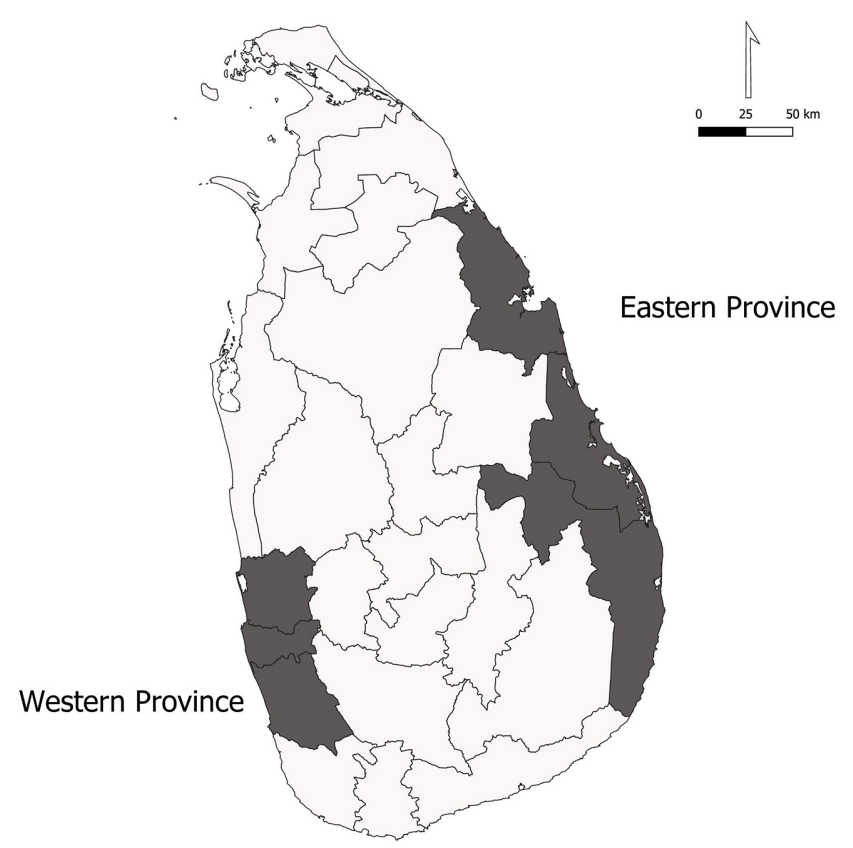

Figure 1 Map of Sri Lanka. b. Establish a shared data platform for Sri Lankan CP surveillance using infrastructure from the Australian CP Register (ACPR).

c. Recruit and train the data collectors to ensure that the data collected is accurate.

2. Use the Sri Lankan CP Register pilot data to describe $\mathrm{CP}$ in the Western and Eastern provinces (figure 1) of Sri Lanka in terms of aetiology, risk factors, CP motor type, functional motor severity, that is, Gross Motor Function Classification System and Manual Ability Classification System and associated impairments.

3. Estimate the prevalence of $\mathrm{CP}$ in 30 Divisional Secretary's Divisions (DS Divisions) in the Eastern and Western provinces using community surveillance.

4. Assess the availability and use of rehabilitation services for children with CP in the Western and Eastern provinces.

5. Describe the strengths and challenges of the community and hospital based surveillance programmes in the Sri Lankan context.

6. Estimate the ongoing resources required for hospital and community based surveillance in the Western and Eastern provinces and to extend CP surveillance to other provinces.

\section{METHODS AND ANALYSIS Case definition}

In this study we will use the approach adopted by the Surveillance of Cerebral Palsy in Europe ${ }^{9}$ and the $\mathrm{ACPR}^{7}$ that allows the use of any definition of CP that includes the following five key elements.

Cerebral palsy:

1. Is an umbrella term for a group of disorders,

2. Is a condition that is permanent but not unchanging,

3. Involves a disorder of movement and/or posture and of motor function,

4. Is due to a non-progressive interference, lesion or abnormality, and

5 . The interference, lesion or abnormality originates in the immature brain. ${ }^{9}$

\section{Inclusion/exclusion criteria}

Any child under 18 years of age who attends the participating hospital sites/community camps and meets the case definition as identified by a suitably qualified professional (consultant level physician in the field of paediatrics, paediatric neurology, paediatric rheumatology, paediatric rehabilitation, developmental and community paediatrics) will be eligible for inclusion. Children under 5 years of age identified by qualified medical personnel as being described as 'at high risk for CP' will be included on the CP register. They will then be reviewed at 5 years of age when a final decision regarding inclusion/exclusion will be made. 


\section{Study locations and populations}

\section{Western Province}

The Western Province has an area of 3654 square kilometres, a population of 5851130 and approximately 90450 live births per year. This province is the most socioeconomically developed part of Sri Lanka, consisting of Colombo, Gampaha and Kalutara districts, 40 DS Divisions and 2505 Grama Niladhari Divisions (GN Divisions).

\section{Hospital-based surveillance}

Participants will be recruited through the three major hospitals in the Western Province. The Lady Ridgeway Hospital is a large tertiary care hospital with more than 900 beds. The hospital serves more than 2500 patients per day and is the largest children's hospital in Sri Lanka. The hospital has paediatric rehabilitation, neurology and orthopaedic specialists and outpatient allied health services which are accessed by children with $\mathrm{CP}$ and their families. The North Colombo Teaching Hospital (NCTH), located in Ragama is a major teaching hospital for the Medical Faculty, University of Kelaniya, and provides a range of tertiary services. The multidisciplinary centre for children with disabilities affiliated with the hospital and situated in the Medical Faculty has nearly 800 registered patients with $\mathrm{CP}$ treated for ambulant care and is the primary training centre for Developmental and Community Paediatrics in Sri Lanka. The NCTH hospital has affiliated specialised rehabilitation services and is one of the few rehabilitation hospitals in Sri Lanka and has rehabilitation specialists. The Colombo South Teaching Hospital is another large tertiary level teaching hospital affiliated with the University of Sri Jayewardenepura, located in Kalubowila, with paediatric neurology services and a multidisciplinary team clinic. Therefore, it is assumed that the majority of children with $\mathrm{CP}$ will access the hospital based services in the Western Province because of the relative ease of accessibility with multiple hospitals providing required services.

We estimate that each year a total of 500 children with $\mathrm{CP}$ access these sites. Therefore, we aim to recruit 800 to 1000 participants from all three settings during the recruitment period which will be completed in June 2022 (figure 2).

\section{Community-based surveillance}

After the hospital-based surveillance has been established in the Western Province, a community based surveillance programme will be launched (figure 2). This will include 15 Divisional Secretariats randomly selected as recruitment regions in the Western Province. Potential participants will be identified and recruited by three community based local public health midwives (PHM) and a research assistant (RA) using a house-to-house survey completed in each of the selected Divisional Secretariats. Potential participants will be invited to attend an assessment and equipment camp for further multidisciplinary assessment by a team of health professionals from CPLF. Children who appear to meet the inclusion criteria for $\mathrm{CP}$ but
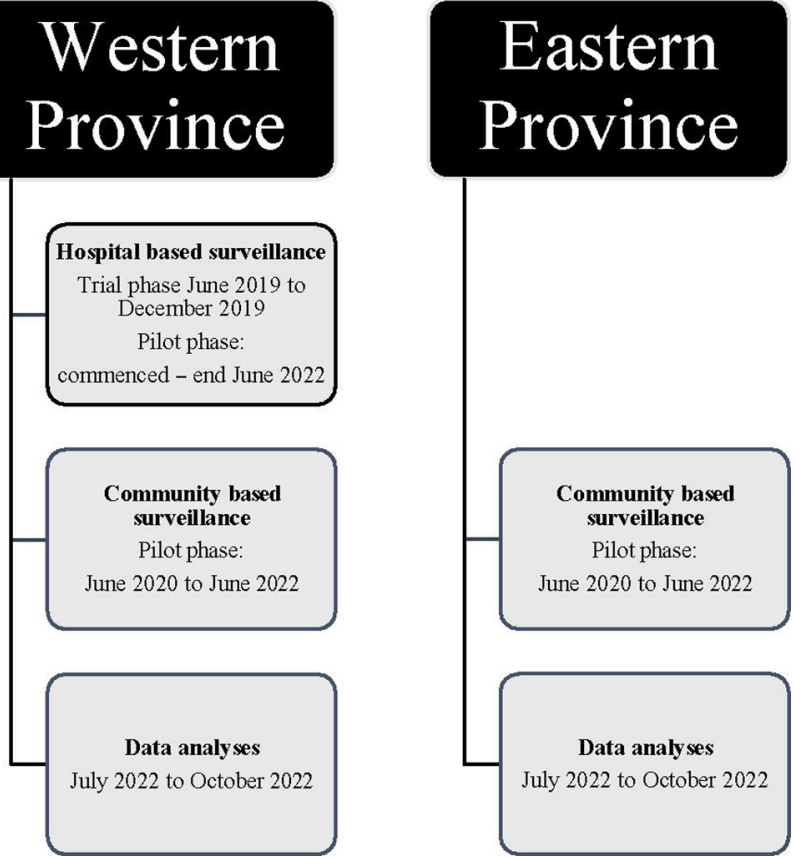

Figure 2 Timeline.

who do not have a formal diagnosis will be referred to the local paediatrician, rehabilitation specialist or neurologist to determine whether they have CP. Coordination between the community and hospital programmes in this province will be required to ensure there are no duplication of registrations. Using available denominator data (live births) and applying a rate of 3.4 per $1000^{7}$ we estimate that this house-to-house survey will identify $>400$ children with CP. The recruitment period will commence June 2020 and finish June 2022, figure 2).

\section{Eastern Province}

The Eastern Province has a population of 1551381 across 9996 square kilometres with approximately 30297 live births per year. This province consists of three administrative districts namely Trincomalee, Batticaloa and Ampara which are further divided into 45 DS Divisions and 1085 GN Divisions. The Eastern Province is the most diverse in Sri Lanka, both ethnically and religiously. This Province has one Base hospital per district but lacks many specialist services such as paediatric rehabilitation and neurology. ${ }^{10}$ The access to services are also difficult in these districts, hence the study is limited to the community-based arm.

\section{Community-based surveillance}

We will randomly select 15 DS Divisions to include 5 DS divisions from each district. Selection of potential participants, recruitment and confirmation of clinical features and diagnosis will take place as per the community-based arm in the Western Province. Potential participants will attend an assessment and equipment camp for further multidisciplinary assessment by a team of health professionals from CPLF. Recruitment will commence in June 2020 and finish in June 2022 (figure 2). Again we estimate $>400$ children will be identified as per the above 
community based sample calculation in the Western Province.

\section{Data set}

In order to allow comparison of findings and/or future research collaboration using Sri Lankan Cerebral Palsy Register (SLCPR) data, the SLCPR Record Form has been developed to include the standard demographic and clinical data fields currently collected by other established CP registers in Australia, Bangladesh and New Zealand (see online supplementary table 1). ${ }^{571112}$ The lead study investigators for the community and hospital surveillance in the Western Province will additionally record total training time per staff member, staffing requirements (number of staff days per week) and any additional expenses associated with undertaking the hospital and community based surveillance programmes.

\section{Data collection \\ Consent}

Participants who may be eligible for inclusion in the register will be identified by health professionals at the hospital sites or by door-to-door surveillance in DS Divisions in the community. Informed consent will be obtained by all families, with information provided in their preferred language (Sinhala, English or Tamil). Consent to participate in the SLCPR includes; (1) permission for the collection, recording and ongoing storage of the SLCPR data, which may include consulting health records, (2) permission to contact health professionals to complete or verify the SLCPR data items and (3) consent to receive invitations to participate in research studies. It is clearly stated in the SLCPR Information Form that no identified data will be reported from the SLCPR.

A record of families who have children who are eligible to participate but choose not to will be maintained to ensure those families are not re-contacted by register staff. Data will be collected by qualified and trained physiotherapist RAs using a tested interviewer administered questionnaire, through family interview, through consultation with the health professional responsible for their care and through review of available health records. Data will be recorded on a SLCPR Record Form. The collected data will be monitored regularly by the senior investigators in terms of completeness and accuracy.

Each participant will be allocated a unique identifier number. SLCPR record forms and data collected at the study sites in the hospital surveillance programme and the community surveillance programme will be securely stored at the Faculty of Medicine, University of Kelaniya. In both the hospital and community surveillance programmes study data forms will only be accessible to study investigators and authorised study staff. Once an SLCPR Record Form has been completed the data will be transferred to the SLCPR online database. The SLCPR uses a customisation of the ACPR database which is maintained in an enterprise-grade facility in Australia. The SLCPR database is backed up on a daily basis and is only accessible to authorised study investigators and nominated delegates with password access.

Training of data collectors

Hospital-based surveillance

Qualified physiotherapists with BSc Honours degrees will be recruited as RAs. They will undergo a 2-month training on clinical skills building, history taking and assessment, communication skills, maintaining ethical standards and recording and storing data. They will collect data for 2 weeks under the supervision of a senior developmental and child disability specialist in pairs to minimise interrater variability in data collection. Any doubts on clinical findings will be cleared during data collection by discussion with available senior clinical specialists in the hospitals.

\section{Community-based surveillance}

RAs with similar educational qualifications to the hospitalbased arm will be recruited. They will undergo basic training and initial trial data collection in the community under the supervision of a qualified clinician. Any questions regarding recording of clinical findings will be cleared by discussion with available senior clinical specialists in the hospitals. This team of RAs will train the PHM to identify eligible participants by using a simple questionnaire on developmental milestones, posture and movement impairments during house-to-house surveys. An RA will accompany the PMHs to confirm the selected children during the house-to-house surveys to recruit for the register. Potentially eligible children without a clinical diagnosis will be referred to the clinical specialists in the hospitals for confirmation.

\section{Data entry}

There are a number of quality control mechanisms in place for data entry. Every data sheet will be checked to highlight any missing data. The family and/or relevant health professional will be contacted with the aim of collecting any missing data. When each data sheet is finalised the data will be entered into the database by an RA. The majority of fields in the database have a limited number of possible responses so unexpected data recorded on the data form will be identified at the point of data entry. Each database record will be independently checked by a second RA against the data sheet.

\section{Data analyses}

We will use descriptive statistics, bivariate analyses $\left(\chi^{2}\right.$ and Fisher's exact test) and effect sizes to compare demographic data between (1) the Western and Eastern provinces and (2) all cases captured in the SLCPR and the general population in Sri Lanka. ${ }^{13}$ We will report the known risk factors, clinical characteristics, associated impairments, access to rehabilitation services in Sri Lanka by province and overall. Descriptive epidemiological measures including an estimated birth prevalence (per 1000 live births, with 95\% CI) of CP for the selected DS Divisions will be estimated from the SLCPR database 
with denominator data sourced from the Department of Census Statistics. ${ }^{12}$

Lastly we will explore data from the Western Province to describe differences in surveillance methodologies. Here we will describe the clinical and demographic characteristics of the CP populations captured and the processes required to complete registrations using the two different methodologies. Total staff and programme costs of each programme will also be calculated.

No individually identifiable information will be included in any reports or future publications. Only aggregated data will be reported and small cell sizes will be reported as $\mathrm{n}<5$ to ensure confidentiality is maintained. Data analysis will be performed using SPSS Statistics software V.24 (SPSS Inc, Chicago, Illinois, USA).

\section{ETHICS AND DISSEMINATION SLCPR Advisory Board}

The SLCPR Advisory Board has been established. This group includes the principal and corresponding investigator, co-investigators from the local group of researchers representing each site of data collection, two members representing the Ministry of Health of Sri Lanka (one designated member from the National Health Research Council and the Programme Manager of the Child Development and Special Needs programme representing the Family Health Bureau of Sri Lanka) and a representative of the College of Paediatricians. This group includes people with expertise in paediatrics, neurology and rheumatology. The SLCPR Advisory Board are the custodians of SLCPR database and will oversee responsible use, maintenance and safe-keeping of all data contained within the SLCPR. The SLCPR Advisory Board will also provide advice on specific regional issues, management of the register, future vision and research requests for identified/de-identified Sri Lankan data, and planning towards establishment of a national database in the future.

\section{Ethics}

Ethical clearance for the SLCPR has been obtained from The University of Kelaniya, National Health Research Council and additional institutional ethics clearances from the relevant hospitals: Ethics Review Committee, Faculty of Medicine, University of Kelaniya (Ref No. P/105/03/2017 and Ref No. P/93/07/2016), Ethics review Committee of Lady Ridgeway Hospital for Children (LRH/DA/05/2018), Ethics Review Committee of Colombo South Teaching Hospital (No. 608), National Health Research Council, Ministry of Health Nutrition and Indigenous Medicine (Ref No. ETR/E/ NHRC/2017).

\section{Dissemination}

To the best of our knowledge this will be the largest study systematically investigating the epidemiology of CP in Sri Lanka. As such it will be important to share research findings with both the Sri Lankan and international community. On completion of this pilot stage we will report the results from each arm of the study by publication of findings in peer-reviewed journals and by presentation at local and international conferences. A summary of findings from the pilot phase will be also made available to all participants through the SLCPR website: http:// srilanka.cpregister.com/.

\section{PATIENT AND PUBLIC INVOLVEMENT}

The development of the SLCPR has been informed by a range of interested and motivated groups. Health professionals working with children with CP in Sri Lanka have been calling for the establishment of a CP register in this country for many years. This study has also been strongly supported by the Ministry of Health, a member of whom has now joined the SLCPR Advisory Board. Members of the CPLF (a community based provider of special education and therapy services), in their multifaceted roles as parents, health professionals and community leaders have been integral to the establishment of the SLCPR and have assisted in guiding the development of the SLCPR Record Form. Our co-author, Mr Gopi Kitnasamy, founder of CPLF, who is the father of a young man with CP, is a member of the SLCPR Advisory Board and heads the community-surveillance arm. Mr Kitnasamy has been integrally involved in the development of the SLCPR from the outset.

\section{IMPLEMENTATION}

The SLCPR database has been developed and tested and the SLCPR Advisory Board has been established and training of data collectors for both the hospital and community based surveillance programmes has been completed. To date $>450$ children with CP have been recruited through the hospital based surveillance programme.

Once the pilot phase of this study has been completed, we will evaluate the feasibility of the SLCPR in relation to both hospital and community based surveillance including recruitment, budget, data management and developing an estimate of the required resources to scale up the current SLCPR programme to include additional provinces.

\section{DISCUSSION}

This will be the first epidemiological study of CP in Sri Lanka. The strengths of this study include the ability to identify differences in the epidemiology and service needs of children with CP living in two different provinces of Sri Lanka. Additionally this project will provide an opportunity to examine both the advantages and challenges of different surveillance methods (hospital and community based). By collecting data in line with other well established CP registers in the region we will have opportunity to view our findings from the SLCPR in the 
context of published data from other $\mathrm{CP}$ registers in both high-income and low-income settings.

Data from the SLCPR will be used to (1) identify rehabilitation needs of children with CP; (2) provide the first estimates of birth prevalence, aetiological risk factors and the clinical profile of CP and (3) inform future plans to expand this register to include all provinces. We expect to disseminate our findings in late 2022.

\section{Author affiliations}

${ }^{1}$ Department of Disability Studies, Faculty of Medicine, University of Kelaniya, Ragama, Western Province, Sri Lanka

${ }^{2}$ Cerebral Palsy Lanka Foundation, Colombo, Western Province, Sri Lanka

${ }^{3}$ Cerebral Palsy Alliance, Discipline of Child and Adolescent Health, Sydney Medical School, The University of Sydney, Sydney, New South Wales, Australia

${ }^{4}$ CSF Global, Dhaka, Bangladesh

${ }^{5}$ Asian Institute of Disability and Development, University of South Asia, Dhaka, Dhaka District, Bangladesh

${ }^{6}$ Discipline of Child and Adolescent Health, Sydney Medical School, The University of Sydney, Westmead, New South Wales, Australia

${ }^{7}$ Central Queensland Public Health Unit, Central Queensland Hospital and Health Services, Rockhampton, Queensland, Australia

${ }^{8}$ Department of Pediatrics, Faculty of Medical Sciences, University of Sri Jayewardenepura, Nugegoda, Western Province, Sri Lanka

${ }^{9}$ Lady Ridgeway Children's Hospital, Colombo, Western Province, Sri Lanka

${ }^{10}$ Department of Pediatrics, Faculty of Medicine, University of Colombo, Colombo, Western Province, Sri Lanka

${ }^{11}$ Grace Centre for Newborn Intensive Care, Sydney Children's Hospital Network, Westead, New South Wales, Australia

${ }^{12}$ Department of Medicine, University of Kelaniya, Kelaniya, Western Province, Sri Lanka

Twitter Thilini Madushika Heiyanthuduwage @htmadushika@gmail.com and Hayley Smithers Sheedy @hss_1

Acknowledgements The authors would like to thank the families of children with CP who are supporting and participating in the SLCPR and would also like to acknowledge the wider SLCPR Group without whom the development of this pilot phase would not be possible. The SLCPR Group includes the author group TMH, SPS, GKi, HSS, GKh, SW, PR, JJ, JW, SM, SG, EW, MM, NM and the following collaborators: Nilanthi de Silva, Shyamani Hettiarachchi, Ranmali Waduge, James Rice, Cathy Morgan, Alicia Spittle and TN Adikari and the representatives from the Ministry of Health.

Contributors All authors (TMH, SPS, GKi, HSS, GKh, RF, SW, JJ, PR, JW, SM, SG, EW, NB, MM and NM) made substantial contributions to the conception and design of this protocol. All authors have read and critically reviewed this manuscript, made revisions and have all approved this final version for submission. All authors agree to be accountable for the future integrity of this study.

Funding This work was supported by Cerebral Palsy Alliance Research Foundation, grant number (PG 7016). HS-S received support through a National Health and Medical Research Council Early Career Fellowship 1144566 and the Australasian Cerebral Palsy Clinical Trials Network.

Map disclaimer The depiction of boundaries on this map does not imply the expression of any opinion whatsoever on the part of BMJ (or any member of its group) concerning the legal status of any country, territory, jurisdiction or area or of its authorities. This map is provided without any warranty of any kind, either express or implied.

Competing interests None declared.

Patient and public involvement Patients and/or the public were involved in the design, or conduct, or reporting, or dissemination plans of this research. Refer to the Methods section for further details.

Patient consent for publication Not required.

Provenance and peer review Not commissioned; externally peer reviewed.

Open access This is an open access article distributed in accordance with the Creative Commons Attribution Non Commercial (CC BY-NC 4.0) license, which permits others to distribute, remix, adapt, build upon this work non-commercially, and license their derivative works on different terms, provided the original work is properly cited, appropriate credit is given, any changes made indicated, and the use is non-commercial. See: http://creativecommons.org/licenses/by-nc/4.0/.

ORCID iD

Thilini Madushika Heiyanthuduwage http://orcid.org/0000-0003-1144-7417

\section{REFERENCES}

1 Rosenbaum P, Paneth N, Leviton A, et al. A report: the definition and classification of cerebral palsy April 2006. Dev Med Child Neurol Suppl 2007;109:8-14.

2 Smithers-Sheedy H, Badawi N, Blair E, et al. What constitutes cerebral palsy in the twenty-first century? Dev Med Child Neurol 2014;56:323-8.

3 Mclntyre S, Morgan C, Walker K, et al. Cerebral palsy-don't delay. Dev Disabil Res Rev 2011;17:114-29.

4 Oskoui M, Coutinho F, Dykeman J, et al. An update on the prevalence of cerebral palsy: a systematic review and meta-analysis. Dev Med Child Neurol 2013;55:509-19.

5 Khandaker G, Muhit M, Karim T, et al. Epidemiology of cerebral palsy in Bangladesh: a population-based surveillance study. Dev Med Child Neurol 2019;61:601-9.

6 Kakooza-Mwesige A, Andrews C, Peterson S, et al. Prevalence of cerebral palsy in Uganda: a population-based study. Lancet Glob Health 2017;5:e1275-82.

7 ACPR. Report of the Australian cerebral palsy register birth years 1995-2012, 2018. Available: https://cpregister.com/

8 Rajindrajith S, Mettananda S, Adihetti D, et al. Neonatal mortality in Sri Lanka: timing, causes and distribution. J Matern Fetal Neonatal Med 2009;22:791-6.

9 Surveillance of Cerebral Palsy in Europe. Surveillance of cerebral palsy in Europe: a collaboration of cerebral palsy surveys and registers. surveillance of cerebral palsy in Europe (SCPE). Dev Med Child Neurol 2000;42:816-24.

10 Annual Health Bulletin. Ministry of health and indigenous services Sri Lanka, 2017. Available: http://www.health.gov.lk

11 Khandaker G, Smithers-Sheedy H, Islam J, et al. Bangladesh cerebral palsy register (BCPR): a pilot study to develop a national cerebral palsy (CP) register with surveillance of children for CP. BMC Neurol 2015;15:173.

12 Goldsmith S, Mclntyre S, Smithers-Sheedy H, et al. An international survey of cerebral palsy registers and surveillance systems. Dev Med Child Neurol 2016;58:11-17.

13 Department of Census and Statistics. Sri Lanka 2019. Available: http://www.statistics.gov.lk/ 